\title{
Central Diabetes Insipidus in an Off-pump Coronary Artery Bypass Grafting Surgery
}

\author{
Prashant Nasa ${ }^{1}$, Girishchandra Varma ${ }^{2}$, Amitabh Kulkarni ${ }^{3}$, Sandeep Chaudhary ${ }^{4}$, Prashant Sagar ${ }^{5}$
}

\begin{abstract}
Polyuria in perioperative coronary artery bypass grafting (CABG) surgery is common and mostly benign. Diabetes insipidus (DI) post-CABG is however very rare and mostly have been related with use of cardiopulmonary bypass (CPB) pump. The patient recovered completely with transient requirement of exogenous vasopressin. The central DI may be considered as a differential diagnosis in case of polyuria in the perioperative period of CABG surgery.

Keywords: Central diabetes insipidus, Complications after coronary artery bypass grafting, Coronary artery bypass surgery, Diabetes insipidus. Indian Journal of Critical Care Medicine (2019): 10.5005/jp-journals-10071-23278
\end{abstract}

\section{Case Description}

A 42-year-old male with a known history of hypertension on tab losartan $50 \mathrm{mg}$ once daily, metoprolol $50 \mathrm{mg}$ once daily, hyperlipidemia on tab atorvastatin $20 \mathrm{mg}$ once daily, chronic smoker (1 pack cigarette/day), admitted with persistent chest pain for 4 hours. The pain was nonradiating, not relived on rest and partial response to nitroglycerine spray. The clinical features, risk factors, and his electrocardiogram (ECG) were showing ST-T changes in lateral leads (I, aVL, V4-V6) with normal troponin I made a diagnosis of unstable angina (USA) - acute coronary syndrome (ACS). His 2D echocardiography (echo) showed regional wall motion abnormality (RWMA) in anterolateral wall with a left ventricle ejection fraction of $55 \%$. He was taken for coronary angiography after standard medical treatment which showed critical ( $\geq 90 \%)$ ostial left anterior descending (LAD) artery and diagonal D1 branch-95\% with left circumflex artery (prominent) mid $90 \%$ stenosis and ramus intermedius small with ostial $90 \%$ lesions. He was referred to cardiac surgeon for urgent CABG. His other laboratory investigations were normal with a serum sodium of $138 \mathrm{mg} / \mathrm{dL}$. The patient was taken for fast-track CABG with off-CPB on beating heart with grafting of left internal mammary artery to LAD and proximal and saphenous vein graft to diagonal-D1 and obtuse marginal. The intraoperative course was eventful with no significant blood loss as cell saver autologous blood recovery system was used. He received $2.1 \mathrm{~L}$ of crystalloid (1.5 L 0.9\% saline and $600 \mathrm{~mL}$ ringer lactate) and urine output was $580 \mathrm{~mL}$ over 4.5 hours. The postoperatively course patient was observed for any bleeding for 3 hours and then extubated same day. His urine output started increasing 5 hours after surgery with around $400-600 \mathrm{~mL} /$ hour. He passed $6.6 \mathrm{~L}$ of urine over 15 hours. His laboratory investigations showed (Table 1) high sodium with relatively low urine osmolality and urine spot sodium was checked which was suggestive of DI. His serum antidiuretic hormone (ADH) was sent, and IV vasopressin was started. The patient urine output progressively decreased to $100 \mathrm{~mL} /$ hour with a dose of 0.01 unit/hour of IV vasopressin and sodium progressively became normal (Fig. 1). Patient was changed to desmopressin spray next day and serial followed with electrolytes and urine osmolality. MRI brain was done which showed no hypothalamic-pituitary macroscopic abnormality. The high serum sodium, low urine osmolality with
${ }^{1}$ Department of Critical Care and ICU, NMC Specialty Hospital, Al Nahda, Dubai, United Arab Emirates

${ }^{2}$ Department of Cardiac Sciences, NMC Specialty Hospital, Al Nahda, Dubai, United Arab Emirates

${ }^{3}$ Department of Nephrology, NMC Specialty Hospital, Al Nahda, Dubai, United Arab Emirates

${ }^{4}$ Department of Endocrinology, Diabetology and Metabolic Disorders, NMC Specialty Hospital, Al Nahda, Dubai, United Arab Emirates

${ }^{5}$ Department of Anaesthesiology, NMC Specialty Hospital, Al Nahda, Dubai, United Arab Emirates

Corresponding Author: Prashant Nasa, Department of Critical Care and ICU, NMC Specialty Hospital, Al Nahda, Dubai, United Arab Emirates, Phone: +971-91-11-25572115, e-mail: dr.prashantnasa@ hotmail.com

How to cite this article: Nasa P, Varma G, Kulkarni A, Chaudhary S, Sagar P. Central Diabetes Insipidus in an Off-pump Coronary Artery Bypass Grafting Surgery. IJCCM 2019;23(11):523-525.

Source of support: Nil

Conflict of interest: None

polyuria and response to IV vasopressin along with low serum ADH, and a clinical diagnosis of central DI was made. The desmopressin spray was reduced from 2 sprays 2 times/day to off spray over 11 days. Patient was followed with serum electrolytes and urine output for 6 months with no recurrence of DI.

\section{Discussion}

Central DI is characterized by polyuria and dilute urine $(<300 \mathrm{mOsm} / \mathrm{kg})$, despite serum hyperosmolality caused by ADH deficiency. ${ }^{1}$ This has been rarely reported after CABG and all these cases were done using $C P B$ pump. Our case is unique as this was off-CPB pump CABG surgery where patient developed central DI postoperatively. ${ }^{2-6}$ CABG is known to cause fluid, electrolyte, and acid-base imbalances; neuroendocrine changes; embolic events besides perioperative bleeding; and cardiac complications. ${ }^{7}$ The fluid and electrolytes alterations are more prominent with use of CPB pump. ${ }^{7}$ ADH is an octapeptide hormone produced in the supraoptic and paraventricular nuclei of the hypothalamus, transported along the hypothalamus-pituitary axis, and stored 
Table 1: Serial trend of significant laboratory investigations

\begin{tabular}{llcc}
\hline Laboratory investigations & Postoperative day 0 & Postoperative day 1 & Postoperative day 6 \\
\hline Serum osmolality $(\mathrm{mOsm} / \mathrm{kg})$ & 341.3 & & 289 \\
Urine osmolality (mOsm/kg) & 98 & 403.9 & 408 \\
Urine sodium (mmol/L) & 32 & & 19 \\
Serum vasopressin $(\mathrm{pg} / \mathrm{mL})$ normal-0.1-4.7 & $<0.1$ & 147 & 138 \\
Serum sodium (mmol/L) & 163 & & \\
Glucose (mmol/L) & 6.09 & 1,980 & 1,860 \\
Urine output & 4,200 & & \\
\hline
\end{tabular}

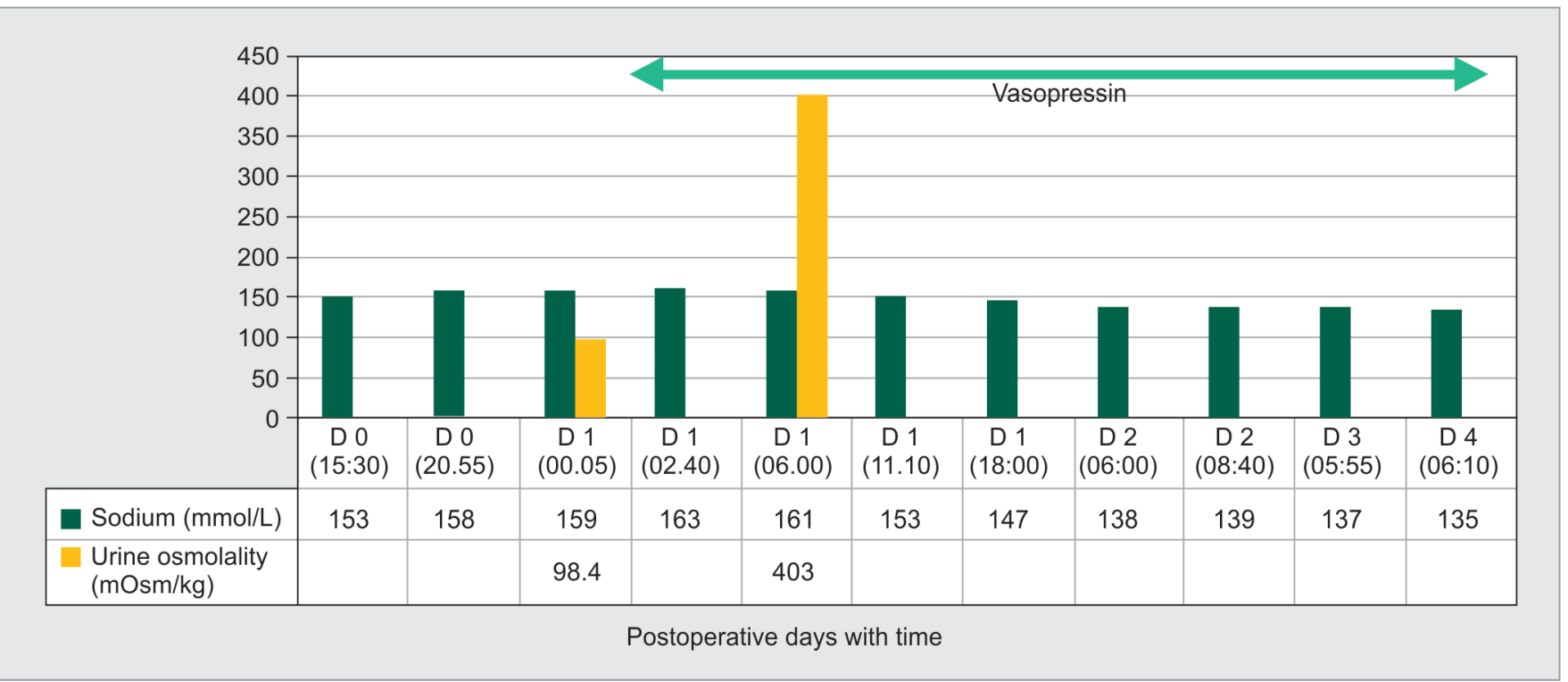

Fig. 1: Trend of sodium and urine osmolality and effect of vasopressin replacement

in the posterior pituitary gland. ${ }^{1}$ The release from the pituitary gland is controlled by nonosmotic or osmotic stimulus. ${ }^{8}$ Osmotic stimulus is mediated by osmoreceptor cells on the front of the hypothalamus which senses the changes in extracellular fluid (ECF) osmolality and control release of $\mathrm{ADH}$. These receptor cells are very sensitive to changes in ECF osmolality and as little as 1-2\% change can trigger release of $\mathrm{ADH}{ }^{8}{ }^{8}$ Nonosmotic control or stimulus is regulated by variation in ECF volume and mediated by low volume receptors in left atrium, aortic arch, and carotid artery. Change in blood volume thus can triggers ADH secretion.

In our patient, the pathophysiology of central DI can be explained by disturbance in either of these two pathways. The transient cerebral ischemia or neurovascular embolic causing focal ischemia is known complications of CABG. ${ }^{7,8}$ There were no episodes of hypotension perioperatively, and postoperative patient neurological status was also without any focal neurological deficits; global ischemia is thus unlikely but microembolic phenomenon to posterior pituitary causing transient dysfunction cannot be ruled out. MRI brain done to rule out any hypothalamic-pituitary pathology was also normal.

There is also possibility of disturbances in nonosmotic stimulus pathway from left atrium which communicate with the hypothalamus through the vagus parasympathetic stimulus. The manipulation of heart for grafting can cause relative left atrial hypovolemia and suppression of ADH release. The central DI after cardiac surgery thus can be associated with disturbances by both or either of stimuli. ${ }^{4}$ In our patient, the other causes of DI and polyuria like fluid overloading, hypokalemia, hypocalcemia, osmotic diuresis, and renal dysfunction was considered and excluded based on the patient's clinical course and laboratory data. ${ }^{1}$ The DI responding to vasopressin replacement with appropriate osmolality and urine output response makes the diagnosis of central DI likely in our patient. In summary, patients who undergo cardiac surgery with or without the use of CPB could rarely develop $\mathrm{DI}$ perioperatively thus significant polyuria should be investigated for possibility of DI along with other differential diagnosis.

\section{References}

1. Verbalis JG. Diabetes insipidus. Rev Endocr Metab Disord 2003;4(2):177-185. DOI: 10.1023/A:1022946220908

2. Uyar IS, Sahin V, Akpinar B, Yurtman V, Abacilar F, Okur FF, et al. Temporary diabetes insipidus in 2 men after on-pump coronary artery bypass grafting. Tex Heart Inst J 2013;40(1):99-101.

3. Kuan P, Messenger JC, Ellestad MH. Transient central diabetes insipidus after aortocoronary bypass operations. Am J Cardiol 1983;52(10):1181-1183. DOI: 10.1016/0002-9149(83)90570-2.

4. Yu CH, Cho JH, Jung HY, Lim JH, Jin MK, Kwon O, et al. A case of transient central diabetes insipidus after aorto-coronary bypass operation. J Korean Med Sci 2012;27(9):1109-1113. DOI: 10.3346/ jkms.2012.27.9.1109.

5. Ekim M, Ekim H, Yilmaz YK, Bolat A. Transient diabetes insipidus following cardiopulmonary bypass. J Coll Physicians Surg Pak 2015;25(Suppl 1):S10-S11. DOI: 04.2015/JCPSP. S10S11. 
6. Ashraf $\mathrm{O}$, Sharif $\mathrm{H}$, Shah $\mathrm{M}$. A case of transient diabetes insipidus following cardiopulmonary bypass. J Pak Med Assoc 2005;55(12): 565-566.

7. Hillis LD, Smith PK, Anderson JL, Bittl JA, Bridges CR, Byrne JG, et al. 2011 ACCF/AHA guideline for coronary artery bypass graft surgery: executive summary: a report of the American College of Cardiology Foundation/American Heart Association Task Force on Practice guidelines. J Thorac Cardiovasc Surg 2012;143(1):4-34. DOI: 10.1016/ j.jtcvs.2011.10.015.

8. Elias LL, Antunes-Rodrigues J, Elias PC, Moreira AC. Effect of plasma osmolality on pituitary-adrenal responses to corticotropin-releasing hormone and atrial natriuretic peptide changes in central diabetes insipidus. J Clin Endocrinol Metab 1997;82(4):1243-1247. DOI: 10.1210/ jcem.82.4.3884. 